\title{
Resúmenes de trabajos libres del $X$ Encuentro Nacional de Medicina del Dormir
}

\section{Abstracts of free works of the X National Meeting of Sleep Medicine}

\section{Integrantes de la Mesa Directiva}

Selene Guerrero-Zúñiga, * Ricardo Bello-Carrera, Gabriel Cruz Zorrilla, ${ }^{\S}$ Alejandro Jiménez-Genchi"

*INER Ismael Cosío Villegas; ${ }^{\circ}$ CMN La RaZa, IMSS; ${ }^{\S}$ Somnoscan, NL, Monterrey; "INP Ramón de La Fuente MuÑIZ

\section{Tratamiento del SAOS con presión positiva continua:} un estudio de costo-efectividad para México

Carrillo Alduenda JL,* Gay-Molina JG,

Pérez-Hernández A, ${ }^{\S}$ Torres-San Miguel GPף

*INER Ismael Cosío Villegas; †Tecnología e Informática PARA LA SALUD; §INFRAMÉdICA; "HGR 1 IMSS

Introducción: El síndrome de apnea obstructiva del sueño (SAOS) es un problema de salud pública y genera cuantiosos gastos, la presión positiva continua en la vía aérea (CPAP) es su mejor tratamiento, pero su costoefectividad no ha sido evaluada en México. Hipótesis: el tratamiento del SAOS con CPAP es costo-efectivo para el sistema de salud mexicano. Objetivo: crear un modelo para evaluar el costo-efectividad del tratamiento del SAOS con CPAP para México. Material y métodos: se creó un modelo de Markov con dos posibles estados de salud: supervivencia o muerte. La posibilidad de transitar se calculó con información nacional e internacional; las premisas del modelo fueron las siguientes: edad promedio 50 años, mortalidad general con tratamiento $0.4 \%$ (misma que en población abierta) y sin tratamiento $20 \%$ (Martí S, 2002), apego a CPAP $80 \%$ (Torre L, 2018), los costos se tomaron de los Grupos Relacionados de Diagnóstico del Instituto Mexicano del Seguro Social. El tiempo de seguimiento fue cinco años. Resultados: el CPAP originó una ganancia de 4.6 años de supervivencia y un ahorro de 36,279 MXN en promedio por paciente con SAOS; además, disminuyó en 4.6 días la hospitalización por complicaciones cardiovasculares, para un servicio que inicia con 300 pacientes y termina con 420, se observó un ahorro significativo a partir del segundo año, llegando a ser de 15'237,304 a los cinco años de tratamiento; el 64\% de los costos fueron generados por complicaciones cardiovasculares. Conclusiones: el tratamiento del SAOS con CPAP es costo efectivo para el sistema de salud mexicano.

Eficacia del dilatador de fosas nasales para disminuir el índice de desaturación de oxígeno (IDO) y T90 comparado con APAP

\author{
Martínez-González O, Orozco-González BN, \\ Hernández-Gordillo Daniel \\ Centro Médico Nacional de Occidente, IMSS
}

Introducción: el síndrome de apnea obstructiva del sueño (SAOS) es un trastorno del dormir ocasionado por el colapso repetitivo de la vía aérea superior durante el sueño, que resulta en desaturación de oxígeno intermitente, despertares y activación simpática. Los dilatadores de fosas nasales (DFN) se comercializan como alternativa en el tratamiento del SAOS; sin embargo, no existe evidencia que respalde su eficacia. Objetivo: comparar la disminución del índice de desaturación de oxígeno (IDO) y del T90 respecto al estudio basal con DFN y con dispositivo de presión positiva automático (APAP) en pacientes con SAOS leve-moderado. Material y métodos: estudio transversal analítico, se incluyeron pacientes con SAOS leve o moderado de la Unidad Metabólica del Centro Médico Nacional de Occidente. Posterior a poligrafía respiratoria se realizó oximetría de pulso en noches consecutivas con dilatador de fosas nasales y con APAP. Los valores de IDO y T90 se presentan con mediana y percentil $25-75$, fueron comparados entre el estudio basal y con la intervención mediante prueba de Wilxocon de rangos con signo. Resultados: se incluyeron 10 pacientes, con $70 \%$ mujeres. Edad media de $47 \pm 11$ años, y media de IMC $44 \pm$ $9.7 \mathrm{~kg} / \mathrm{m}^{2}$. La poligrafía inicial presentó un IAH 14.4/h (11-22.9), IDO $29.2 / \mathrm{h}$ (21.7-35.6) y T90 78\% (18-88). Con DFN el IDO fue $24.6(20.5-38.1, \mathrm{p}=0.18)$ y un T90 de $72 \%(15-87, \mathrm{p}=0.18)$. Con APAP el IDO 11.8/h (5.4-20.4, p < 0.05) y T90 24\% (3-67\%, p < 0.05). Conclusiones: en pacientes con apnea leve-moderada el uso de dilatadores de fosas nasales no es eficaz para reducir el ODI o T90. El APAP disminuyó significativamente ambos parámetros; sin embargo, el T90 en promedio fue de $24 \%$, las causas de esto escapan al propósito del presente estudio.

\section{Características del sueño en niños con trastorno del espectro autista}

\author{
Ayala-Guerrero F \\ FACULTAD DE PSICOLOGíA, UNAM
}

Introducción: el trastorno del espectro autista (TEA) es un desorden heterogéneo del neurodesarrollo definido conductualmente, estos pacientes pueden presentar también alteraciones comórbidas como epilepsia hasta en un tercio de los casos y ansiedad. Estudios llevados a cabo por medio de cuestionarios muestran varios problemas de sueño en pacientes pediátricos con TEA. Sin embargo, hay relativamente pocos estudios llevados a cabo con polisomnografía. La obtención de registros de sueño es importante porque permite detectar actividad epileptiforme. Objetivo: describir las características del sueño y detectar anormalidades de EEG en pacientes con TEA. Material y métodos: se llevaron a cabo regis- 
tros polisomnográficos durante dos noches consecutivas en niños del sexo masculino con TEA y en niños control de edad similar (ocho a 12 años). La primera noche fue considerada de habituación, comparándose únicamente los datos obtenidos de la segunda noche. Se obtuvo la eficiencia de sueño, así como los porcentajes de cada fase del sueño de los dos grupos. Se analizaron y compararon las características del EEG. Resultados: los niños con TEA presentaron menor eficiencia de sueño, sueño fragmentado y disminución del sueño delta y MOR. Se observó actividad cerebral epileptifome en el 50\% de los niños con TEA. Conclusiones: los niños con TEA presentaron alteraciones cuantitativas y cualitativas del sueño, así como anormalidades del EEG.

\section{Revisión sistemática del desempeño diagnóstico del monitor portátil tipo IV versus polisomnografía para diagnóstico de síndrome de apnea obstructiva del sueño en niños}

Portillo-Vásquez AM, Santamaría-Aldaco J, Guerrero-Zúñiga S

$$
\text { INER ISMAel Cosío Villegas }
$$

Introducción: el síndrome de apnea obstructiva del sueño (SAOS) se define por episodios repetidos de reducción o cese del flujo respiratorio por obstrucción parcial o total de la vía aérea superior durante el dormir. La prevalencia en niños se ha reportado de 1 a $4 \%$. El estándar de referencia para diagnóstico es la polisomnografía en laboratorio de sueño (PSG), por su costo y complejidad está poco disponible. Los monitores portátiles tipo IV cuentan con uno a tres canales y pueden utilizarse en casa. No hay una revisión sistemática que analice el desempeño de los monitores tipo IV contra polisomnografía para el diagnóstico de SAOS en niños. Objetivo: conocer el desempeño diagnóstico de los monitores portátiles tipo IV para el diagnóstico de SAOS en niños sin comorbilidades. Material y métodos: se realizó una búsqueda sistemática en diversas bases de datos (MEDLINE, LILACS, Web of Science, biblioteca COCHRANE, etc.) de estudios con criterios de inclusión (monitor tipo IV contra polisomnografía para diagnóstico de SAOS en niños) y exclusión (comorbilidades). Se evaluaron los títulos y resúmenes, de cumplir criterios se revisó el texto completo. Resultados: se revisarón 44 estudios. La gran mayoría analizan oximetría. En 24 estudios evaluaron un monitor independiente y 20 subanálisis de señales obtenidas en PSG. En 32 estudios se analizó oximetría sola, cinco evaluaron oximetría junto con un canal extra, cinco un sensor de movimiento, dos audio, uno video y uno variabilidad del electrocardiograma. Conclusiones: los datos obtenidos en esta revisión no nos permiten realizar una recomendación favorable al uso de los monitores tipo IV en niños. El $45 \%$ de los estudios reportan subanálisis de PSG que resta validez para su consideración como alternativa de diagnóstico.

\section{Parasomnia no especificada en paciente adulto mayor: Reporte de caso}

Barrera-Medina A, Galicia-Polo L, Gutiérrez Escobar R, Mendoza Cáceres L, Muñoz Hernández G

Clínica de Trastornos del Sueño, unAM

Introducción: las parasomnias son conductas indeseables que ocurren al inicio, durante o al despertar del sueño. En relación a las parasomnias de inicio en el sueño No MOR incluyen despertares confusionales, sonambulismo y terrores nocturnos; son frecuentes en niños, disminuyendo su incidencia en adultos. Las parasomnias de activación en adultos se asocian a trastornos psiquiátricos como depresión y/o ansiedad. Objetivo: describir el caso clínico de una paciente adulta mayor con parasomnias del sueño No MOR. Material y métodos: descripción de un caso clínico. Resultados: paciente femenino de 64 años de edad, con antecedente de enuresis nocturna de los siete a los ocho años de edad, acude a la Clínica de Sueño por presentar desde hace 17 años conductas de deambular fuera de la cama caracterizadas por buscar a una persona, con gritos, además de amnesia, las cuales desaparecieron por un período de cinco años y reaparecen hace dos años con una frecuencia de cinco eventos por semana, hasta tres ocasiones por noche, se ha despertado en lugares diferentes de su hogar. Hace dos años presenta insomnio combinado relacionado a preocupaciones constantes. La exploración neurológica es normal, el Inventario de Ansiedad de Beck es de 20 puntos. EEG: disfunción generalizada, resonancia magnética: hiperintensidades puntiformes dispersas (glióticos) en sustancia blanca subcortical, pérdida del volumen cortico-subcortical. Se le realizó PSG 10/20, presentó un IAH de 10.5/hora de sueño, así como parasomnia de activación con despertares confusionales. Diagnósticos: insomnio comórbido a trastorno de ansiedad generalizada, parasomnias de activación, SAHOS leve. Se le indicó tratamiento a base de escitalopram $10 \mathrm{mg}$ al día e higiene de sueño. Tres meses posteriores al tratamiento, el Inventario de Ansiedad de Beck disminuyó a 10 puntos, ya no ha presentado conductas durante el dormir. Conclusiones: paciente que presenta sonambulismo y despertares confusionales asociados estrechamente a trastorno de ansiedad generalizada.

\section{Sintomatología clínica de trastorno de conducta de sueño MOR en pacientes menores de 50 años con uso de antidepresivos}

\author{
Aedo-Torrado A, Alatriste-Booth $\mathrm{V}$ \\ instituto Nacional de Neurología y Neurocirugía \\ «Manuel Velasco Suárez»
}

Introducción: el trastorno de conducta de sueño MOR (TCSM) es una parasomnia caracterizada por conductas nocturnas disruptivas acompañadas por sueños vividos con pérdida de la atonía típica de la etapa MOR. Su prevalencia en población general es de $0.38-2.1 \%$, siendo mayor en pacientes con Parkinson y otras sinucleinopatías. Aproximadamente $81 \%$ de los pacientes con TCSM clasificados como idiopático eventualmente desarrollaron algún tipo de sinucleinopatía. Se ha reportado asociación del TCSM con el uso de antidepresivos. Objetivo: describir la prevalencia de síntomas de TCSM en menores de 50 años con uso de antidepresivos. Identificar rasgos y comorbilidades asociados al desarrollo del TCSM. Material y métodos: transversal prospectivo de pacientes < 50 años con uso de antidepresivos en el Intituto Nacional de Neurología y Neurocirugía. Se aplicó el cuestionario de detección del TCSM (RBDSQ) en español. Un resultado $\geq 5$ fue considerado positivo a TCSM. Se realizó estadística descriptiva de los datos recabados, así como análisis univariados y multivariados con regresión binomial en el programa SPSS versión 23.0. Resultados: se incluyeron 68 pacientes, en su mayoría mujeres $(77.9 \%)$, con una edad media de $35.1 \pm$ 
8.7 años. El trastorno depresivo mayor (51.5\%) y de ansiedad (29.4\%) fueron los dos principales diagnósticos por los que se indicaron antidepresivos. La prevalencia de TCSM fue de $60.3 \%$. De los cuales $37(90.2 \%)$ fueron mujeres y cuatro $(9.8 \%)$ fueron hombres $(p=0.003)$. El único factor independiente asociado con síntomas de TCSM fue el género femenino $(\mathrm{p}=0.003)$; mientras que la edad, lateralidad manual, tabaquismo, ingesta de alcohol, toxicomanías, sedentarismo, hipertensión, diabetes mellitus, el tipo de antidepresivo, fármacos asociados y diagnóstico principal no demostraron asociación significativa. Conclusiones: se encontró una alta prevalencia $(60.3 \%)$ de sintomatología clínica de TCSM en pacientes $<50$ años con uso de antidepresivos. El género femenino confirió un riesgo mayor de presentar estos síntomas. Estudios de cohorte con confirmación de TCSM por polisomnografía y seguimiento para detección de sinucleinopatías son necesarios para confirmar esta asociación y esclarecer si se trata de un efecto secundario del medicamento o si la medicación desenmascara síntomas de un padecimiento subyacente.

\section{Síntomas de sueño y comorbilidades de los pacientes con sospecha de apnea de sueño referidos a estudio diagnóstico simplificado}

\author{
Colonia-Cano CJ, ${ }^{*}$ Che-Morales JL, ${ }^{\ddagger}$ Guerrero-Zúñiga $S^{\S}$ \\ *IMSS, HGR1, YUCATÁN; \#IMSS, UMAA, YUCATÁN; \\ \$INER ISMAEL COSIOO VILLEGAS
}

Introducción: El síndrome de apnea obstructiva del sueño (SAOS) afecta al $14 \%$ de los hombres y al $5 \%$ de las mujeres. Los principales síntomas son ronquido, apneas presenciadas, somnolencia diurna excesiva, fatiga e incrementa el riesgo para enfermedad cardiovascular y alteraciones metabólicas. Los estudios simplificados son accesibles y permiten el diagnóstico en pacientes sintomáticos bien seleccionados. Objetivo: describir los síntomas de sueño, comorbilidades y uso de fármacos en los pacientes referidos por sospecha de SAOS, adicionalmente describir la prevalencia de hipoxemia relacionada a sueño y factores asociados. Material y métodos: estudio retrospectivo. Se analizaron de forma consecutiva pacientes enviados por sospecha de SAOS; se realizaron escalas de predicción e interrogatorio de síntomas, comorbilidades y uso de fármacos. El diagnóstico se confirmó mediante polígrafo Stardust II. Se definió SAOS como un RDI $\geq 15$ eventos/hora. Y fueron titulados de manera automática. Las variables categóricas fueron reportadas como frecuencias y porcentajes, las continuas con mediana (P25-P75), se compararon los pacientes con o sin hipoxemia durante el sueño con $\chi^{2}$ o U- Man Whitney según el tipo de variable. Resultados: En el período de agosto 2016 a abril de 2018 se estudiaron 1,436 pacientes, $51.4 \%$ hombres, $74.3 \%$ obesos. El $44.1 \%$ presentaban $\geq 2$ comorbilidades. El $6.1 \%$ usaban benzodiazepinas. El $57.8 \%$ presentó apnea grave y el $61.4 \%$ con hipoxemia relacionada al sueño. El síntoma más prevalente es el ronquido. El reporte de apneas presenciadas es mayor en aquellos con hipoxemia $(\mathrm{p}<0.01)$. Todas las variables de poligrafía respiratoria reflejan mayor gravedad en el grupo con hipoxemia y requieren mayor presión terapéutica. Conclusiones: los síntomas más frecuentes son el ronquido y la fatiga, la comorbilidad más frecuente es la HAS, neumopatía crónica y enfermedades metabólicas, más de la mitad de la población tiene hipoxemia durante el sueño y éstos requieren presiones terapéuticas mayores.

\section{Valoración del efecto del Dispositivo de Avance Mandibular en la vía aérea superior mediante IRM}

\author{
Gutiérrez-Vargas R, ${ }^{*}$ Hidalgo-Tobon S, ${ }^{*}$ García-Campos E, ${ }^{*}$ \\ Morón-Mendoza A, ${ }^{\ddagger}$ Ruiz-Morales $M,{ }^{\S}$ Miranda-Casarrubia $M$, ${ }^{*}$ \\ Jiménez-Correa $U,{ }^{*}$ Gutiérrez-Escobar $\mathrm{R},{ }^{*}$ \\ Azpiroz-Leehan J, Santana-Miranda R* \\ *Clínica de Trastornos del Sueño, UnAM; ₹Centro Nacional \\ de InVEstigación en Imagenología e InStRumentación MÉdicA; \\ §CMN 20 DE NOVIEMBRE, ISSSTE
}

Introducción: el uso de dispositivos de avance mandibular (DAM) ha demostrado ser útil en el síndrome de apnea/hipopnea obstructiva del sueño (SAHOS). Debido a las características anatomo-funcionales de la vía aérea superior, su relación con el DAM es compleja y poco estudiada. Hipótesis: los DAM incrementan los espacios retropalatino, retrolingual y retroepiglótico. Objetivo: evaluar el efecto de los DAM en el diámetro de la vía aérea superior en tres niveles, utilizando imágenes de resonancia magnética (IRM). Material y métodos: participaron 14 pacientes con diagnóstico de SAHOS asociado a base de lengua amplia y obstructiva. Se realizó un DAM personalizado para cada paciente, posteriormente se realizaron IRM con equipo de 3 Tesla, Philips, con y sin el DAM en decúbito dorsal y en vigilia. Para las mediciones se utilizó el software OSIRIX MD, sobre el corte medio sagital de $2 \mathrm{~mm}$. Resultados: el espacio que más se amplió fue el retroepiglótico $(n=9)$, seguido del retropalatino $(n=5)$ y del retrolingual $(n=4)$, sin encontrar diferencias estadísticas significativas con respecto a las mediciones con y sin la aplicación del DAM. Sin embargo, se encontró el cambio en las mediciones de volumetría realizadas a nivel del aérea estudiada, se aplicó la prueba estadística t Student a dos colas con un valor 0.426. Conclusiones: el efecto del DAM sobre el SAHOS es debido al incremento del espacio de la vía aérea superior por un efecto volumétrico como lo muestra la valoración mediante IRM. Consideramos estudiar dicho efecto en las etapas del sueño.

\section{Apnea del sueño en pacientes con infarto cerebral crónico}
Valdés-Galván RE, ${ }^{*}$ Alatriste-Booth $\mathrm{JV},{ }^{*}$ Arauz-Góngora $\mathrm{A}$, Escobar-Alvarado J, ${ }^{\ddagger}$ Sandoval-Zárate J, ${ }^{\ddagger}$ Vargas J*
*Instituto Nacional de Neurología y Neurocirugía "Manuel Velasco"; ;instituto Nacional de Cardiología

Introducción: la enfermedad vascular cerebral (EVC) comprende distintas enfermedades de la irrigación cerebral que alteran su funcionamiento, sea por oclusión arterial, venosa o ruptura arterial. Es prevenible con factores de riesgo bien identificados. $\mathrm{La}$ apnea del sueño es uno de dichos factores. Comprende un rango de alteraciones que afectan el patrón respiratorio durante el sueño con impacto subjetivo y objetivo en el paciente. Asimismo, mediante distintos mecanismos el infarto cerebral predispone a apnea de sueño. Se han encontrado prevalencias de entre 70-90\% de apnea del sueño en los primeros meses tras el infarto cerebral. Existe poca información sobre si dicha prevalencia disminuye conforme evoluciona el infarto o si su presencia es predecible con escalas clínicas. Hipótesis: existe una diferencia en la prevalencia de SAOS en los pacientes con infarto cerebral de seis meses a un año de evolución, 
contra aquellos $<6$ meses de evolución según cohortes históricas de al menos 50\%. Objetivo: evaluar la proporción de pacientes con EVC de 6-12 meses de evolución que cumplen criterios para apnea del sueño por poligrafía respiratoria. Comparar dicha proporción con estudios previos con pacientes con evolución menor a seis meses. Evaluar el desempeño de las escalas STOP-BANG, Epworth y Berlín en la predicción de apnea del sueño. Material y métodos: estudio prospectivo realizado en 31 pacientes con EVC de seis meses a un año de antigüedad en dos centros; se aplicaron los cuestionarios antes mencionados y poligrafía, con registro de al menos cuatro horas. Se comparó la prevalencia encontrada versus la histórica prueba binomial de igualdad. Resultados: $77 \%$ de los pacientes cumplieron criterios de apnea del sueño. Siete pacientes presentaron respiración de Cheyne-Stokes. Ocho pacientes no tuvieron cuestionario predictivo positivo. Conclusiones: la prevalencia de apnea en EVC entre 6-12 meses no es distinta a la reportada en pacientes menor tiempo de evolución.

\section{Titulación remota de ventilación mecánica no invasiva nocturna: resultados preliminares}

Velarde-Calleros JL, Torres-Fraga MG; Vargas-Becerra M, Carrillo Alduenda JL

INER ISMAEL Cosío VILLEGAS

Introducción: el estándar para titular una ventilación mecánica no invasiva (VMNI) es un procedimiento manual a través de polisomnografía, pero las nuevas herramientas de telemedicina podrían ahorrar tiempo, costos y mejorar apego. Hipótesis: la titulación remota de VMNI en pacientes con hipoventilación es factible y efectiva. Objetivo: evaluar la factibilidad y efectividad de realizar una titulación remota de VMNI en pacientes con hipoventilación durante el sueño. Material y métodos: a pacientes adultos con hipoventilación durante el sueño se les colocó un equipo BinivelAirCurve-ST-ResMed con mascarilla oronasal y fueron monitorizados a través de la nube Airview. La programación inicial fue: EPAP $4 \mathrm{cmH}_{2} \mathrm{O}$, IPAP $8 \mathrm{cmH}_{2} \mathrm{O}$ y frecuencia respiratoria 2 menor a la basal; las valoraciones fueron los días 3, 6, 14, 21 y 28; los objetivos fueron una P95 de volumen corriente (VC) de $10 \mathrm{~mL} /$ kg-peso-ideal y un IAH residual menor a $10 \mathrm{~h}^{-1}$; EPAP e IPAP se incrementaron $2 \mathrm{cmH}_{2} \mathrm{O}$ en presencia de apneas obstructivas y la IPAP $2 \mathrm{cmH}_{2} \mathrm{O}$ en caso de no alcanzar VC objetivo. Resultados: se incluyeron tres pacientes, hombres con deformidad torácica; en dos de los tres pacientes se cumplieron los objetivos de volumen corriente e IAH observándose una normalización de la gasometría. Las horas de uso promedio fueron entre 5:13 y 8:25 h. Las molestias referidas: sequedad nasofaríngea, fuga, incomodidad, marcas o irritación facial. Los tres pacientes refirieron estar dispuestos a seguir utilizando el equipo y reportaron descansar mejor, más energía y menor disnea. Conclusiones: la titulación remota de una VMNI a través de la nube es factible y podría ser efectiva.

\section{Control de SAOS grave mediante el uso de dispositivo de avance mandibular (DAM mex). Caso clínico}

García-Campos EP, Galicia-Polo L, Ruiz-Morales M, Gutiérrez-Vargas R, Bernal-Bermúdez R, Valiente G. Santana-Miranda R

Clínica de Trastornos del Sueño, UNAM
Introducción: el manejo multidisciplinario del DAM en el control del SAOS ha demostrado eficacia en el tratamiento de ronquido y SAOS. La recomendación actual es para ronquido y SAOS leve a moderado (IMC $<25 \mathrm{Kg} / \mathrm{m}^{2}$ ); la clínica muestra que la selección de casos está basada en la valoración clínica, complementada con pruebas endoscópicas y radiográficas, así como en la selección personalizada del DAM. Objetivo: valorar cambios polisomnográficos con el uso de DAM en SAOS grave. Material y métodos: reporte de un caso clínico. Resultados: paciente masculino de 52 años acude por presentar ronquido intenso, somnolencia subjetiva (Epworth $=12)$. La exploración muestra obesidad grado I (IMC $31 \mathrm{~kg} / \mathrm{m}^{2}$ ), hipertrofia de cornetes, Mallampati IV, base de lengua amplia y presencia de ronquido nasal y orofaríngeo. $\mathrm{SACS}=45$ puntos. La polisomnografía reporta SAOS grave $(\mathrm{IAH}=34.3 \mathrm{ev} / \mathrm{h})$. Indicando semana terapéutica con APAP marca Resmed ${ }^{\circledR}$ programado de 4 a $20 \mathrm{cmH}_{2} \mathrm{O}$, mascarilla nasal, seguido de CPAP a 10 $\mathrm{cmH}_{2} \mathrm{O}$ durante un mes, mostrando escasa mejoría e intolerancia al CPAP, por lo que se empleó un DAM de tipo personalizado (DAM mex). En el seguimiento se observan cambios subjetivos (disminución de ruido de ronquido, somnolencia diurna y sueño reparador). Los efectos temporales con DAM mex fueron salivación y dolor dental. Posterior a tres meses se realizó poligrafía (STARDUST II Respironics ${ }^{\circledR}$ ) con DAM, observando disminución en el IAH (34.3 a $17.8 \mathrm{ev} / \mathrm{h}$ ), eventos de ronquido (1854 a $111 \mathrm{ev)}$ y $\mathrm{SaO}_{2}(91 \%$ a 93\%). El paciente accede a retomar el CPAP utilizando DAM mex, como alternativa conjunta, refiriendo mayor comodidad y mejor calidad de sueño. Conclusiones: el uso de DAM mex resultó ser eficaz al mejorar los síntomas y disminuir los eventos respiratorios durante el sueño. El DAM puede ser una buena alternativa en forma individual o en conjunto con otros tratamientos como el CPAP aún en SAOS grave.

\section{Diferencias sexuales en la organización temporal de la actividad eléctrica cerebral en pacientes con síndrome de apnea obstructiva del sueño}

\author{
Muñoz-Torres Z, Santana-Miranda R, Galicia-Polo L, \\ Gutiérrez-Escobar R, Jiménez-Correa $U$ \\ Clínica de Trastornos del Sueño, UnAM
}

Introducción: el síndrome de apnea obstructiva del sueño (SAOS) está asociado con síntomas nocturnos y diurnos. Se ha identificado consistentemente mayor predominancia y severidad en el sexo masculino; sin embargo, la integridad estructural de la materia blanca está más afectada en el sexo femenino. Actualmente, no hay investigaciones previas que estudien las diferencias sexuales en la actividad eléctrica cerebral en pacientes con SAOS. Hipótesis: existen diferencias significativas entre ambos sexos en la actividad oscilatoria cerebral en pacientes con SAOS. Objetivo: comparar la organización temporal de la actividad eléctrica cerebral, durante el sueño y la vigilia, en pacientes con SAOS moderado a grave. Material y métodos: estudio transversal analítico, se incluyeron 45 pacientes (22 mujeres) se les hizo un estudio polisomnografico (PSG) en polisomnografos Cadwell, Easy 3 en la Clínica de Trastornos del Sueño de la Facultad de Medicina de la UNAM. Se comparó la densidad del poder espectral $(1-50 \mathrm{~Hz})$ entre los sexos y gravedad del SAOS. Las pruebas estadísticas se hicieron con el programa SPSS versión 16; el estudio contó con aprobación del Comité de 
Neumol Cir Torax. 2019; 78 (4): 404-408

Ética e Investigación. Resultados: respecto a las mujeres, los pacientes del sexo masculino tuvieron menor densidad de poder de las frecuencias rápidas; los pacientes con SAOS grave tuvieron menor poder sigma durante el sueño No MOR y mayor poder delta durante el sueño MOR. Sin embargo, en el sexo femenino no se identificaron diferencias al comparar la densidad de poder entre los grupos de apnea moderada contra grave. El grupo de apnea grave se caracterizó por menor poder de la banda beta durante la vigilia y mayor poder de delta en MOR. Conclusiones: aunque el diagnóstico es el mismo para ambos sexos, las diferencias fisiológicas entre hombres y mujeres también provocan cambios significativos en la actividad oscilatoria cerebral.

Correspondencia:

Dra. Selene Guerrero-Zúñiga

Correo electrónico: seleguerrero85@gmail.com 